\title{
Patients repeatedly attending accident and emergency departments seeking psychiatric care
}

\author{
Eugene F. Okorie, ${ }^{1}$ Colm McDonald, ${ }^{1}$ Brendan Dineen ${ }^{1}$
}

The Psychiatrist (2011), 35, 60-62, doi: 10.1192/pb.bp.108.024455

${ }^{1}$ National University of Ireland, Galway Correspondence to Eugene F. Okorie (eugene.okorie@nuigalway.ie)

\begin{abstract}
Aims and method This study was conducted to ascertain the clinical characteristics of patients who repeatedly attend accident and emergency (A\&E) department seeking psychiatric assessment and care. We identified 24 individuals who attended frequently and compared them with 72 matched single attenders using data abstracted by chart review.

Results Individuals who attended frequently made up $5.3 \%$ of all patients who attended the A\&E department for psychiatric care but accounted for $19 \%$ of all such presentations. Compared with controls, they were younger at first psychiatric contact and had higher rates of schizophrenia and psychiatric admissions.
\end{abstract}

Clinical implications A small but distinctive group of patients repeatedly seek and obtain hospital-based psychiatric care. Early identification of these patients and targeting them with effective, community-oriented strategies, such as home treatment, could improve their quality of life and prognoses as well as reduce the cost of their care.

Declaration of interest None.
Attendance at accident and emergency (A\&E) departments by patients seeking psychiatric care is quite common as $\mathrm{A} \& \mathrm{E}$ departments usually provide crisis intervention as well as serve as an entry point for hospital admission., However, some patients repeatedly attend A\&E seeking assessment and psychiatric care. These patients may have unmet mental health needs that require more service input. Identifying the clinical characteristics of this patient group and targeting them with more suitable care strategies may reduce such frequent visits to the A\&E department.

Individuals who frequently attend $\mathrm{A} \& \mathrm{E}$ for psychiatric care have been characterised by other studies ${ }^{3,4}$ as being younger males, of Black and minority ethnic background, unmarried, unemployed, and being more likely to have diagnoses of personality disorders and substance misuse. The aim of our study was to identify the clinical characteristics of patients who frequently attend for emergency psychiatric assessment and treatment at the A\&E department of the University College Hospital in Galway, Ireland. Emergency psychiatric care at the hospital is shared between a psychiatric emergency liaison team and the out-of-hour duty psychiatric doctors. Ongoing care is provided in the community or acute psychiatric unit by various multidisciplinary teams.

We identified patients who received emergency psychiatric care in the $\mathrm{A} \& \mathrm{E}$ department three or more times in a 6-month period and compared their demographic and clinical characteristics with those who received such care only once in the same period. We also examined the relationships between frequent $\mathrm{A} \& \mathrm{E}$ attendance for psychiatric care and hospital admission.

\section{Method}

All patients who presented to the A\&E department seeking emergency psychiatric care from 1 July to 31 December 2007 were identified. The number of times each patient had attended over that period was tabulated and those who attended frequently were identified.

Frequent attenders were defined as those patients who presented for psychiatric care three or more times, whereas single attenders accessed psychiatric care only once within this 6-month period. The threshold of three visits was selected as being likely to represent a subgroup of patients with higher than average visiting profile. Three or more visits were in excess of the 95th percentile for attendances to the A\&E department for psychiatric care.

Each frequent attender was compared with three randomly selected single attenders matched by age (5-year age bands) and gender. Random selection of the single attenders was based on computer-generated random numbers. In all, 26 frequent attenders were identified; however, data were available for only 24 of them (92.3\%). These were then matched with 72 single attenders. The psychiatric records of the 96 individuals were then examined. The information collected for each patient included: 
- patient's demographic details, diagnosis, use of psychiatric services and psychotropic medications (prescribed as of 31 December 2007)

- number and length of admissions in the period between 1997 and 2007 (records for admissions before 1997 were not available).

The study was viewed as a service evaluation and therefore did not require ethical approval or patient consent. Confidentiality and anonymity of all patients' data were preserved throughout.

Statistical analysis was carried out using SPSS version 15 for Windows. Descriptive statistics were reported as frequencies, percentages and means. Comparisons were made between frequent and single attenders using Student's $t$-test for continuous normal data, Mann-Whitney test for non-normal data and $\chi^{2}$-tests for categorical data.

\section{Results}

Between 1 July and 31 December 2007, 489 patients, aged 14-94 years with a mean age of 36.7 years (s.d.=14.2), attended the A\&E department 639 times seeking psychiatric assessment and care. Of these patients, 409 attended once (83.6\%); 54 twice (11.1\%); and 26 three or more times (5.3\%). The last group contributed to $19 \%$ of all A\&E attendances. Frequent and single attenders were comparable in terms of living situation, marital status and employment status (Table 1). In terms of age, for both frequent and single attenders the mean was 36 years (s.d. =10), and gender distribution was identical in both groups: 12 male and 12 female frequent attenders, and 36 male and 36 female single attenders.

Frequent attenders had longer psychiatric histories compared with single attenders (15 v. 9 years), with a mean age at first psychiatric contact for the frequent attenders of 21.23 years (s.d. $=4.45)$ compared with 26.70 years (s.d. $=8.60)$ for single attenders $(t=-3.230, P=0.003,95 \%$ C.I -8.899 to -2.033 , unequal variance version of the Student's $t$-test). Frequent attenders were also more likely than single attenders to have schizophrenia $(P=0.03)$ (Table 2).

As of 31 December 2007, 19 frequent attenders (79.2\%) and 44 single attenders (62.9\%) were prescribed psychotropic medications. Of these, 16 frequent attenders (66\%) were prescribed antipsychotics compared with 28 single attenders $(38.9 \%)(P=0.012)$.

Frequent attenders were significantly more likely to have been admitted into the acute unit or day hospital than single attenders $(P=0.03$ and $P=0.05$ respectively) (Table 3 ).

Frequent attenders were found to have a shorter mean interval between hospital discharges to the next A\&E presentation (10.9 days; 95\% CI 3.8 to 17.9) than single attenders (21.3 days; $95 \% \mathrm{CI}-5.3$ to 47.8 ). The median interim between discharge and A\&E presentation for these two groups differed ( 6 days for frequent attenders $v .9 .5$ days for single attenders). However, the mean rank difference for frequent and single attenders (11.8 v. 12.3 days, respectively) for this interval was not statistically significant (MannWhitney $U=57.5, P=0.87$ ).

Frequent attenders also had a shorter mean interval between presentation to the $\mathrm{A} \& \mathrm{E}$ department and

Table 1 Demographic characteristics of the study
population
\begin{tabular}{llll}
$n$ & $n(\%)$ \\
\cline { 2 - 4 } & $\begin{array}{l}\text { Frequent } \\
\text { attenders }\end{array}$ & $\begin{array}{c}\text { Single } \\
\text { attenders }\end{array}$ & $\chi^{2}$ \\
\hline Single/separated/divorced & $16(67)$ & $54(75)$ & 0.6 \\
\hline Unemployed $^{\text {a }}$ & $19(83)$ & $49(75)$ & 0.5 \\
\hline Lives alone $^{b}$ & $1(4.8)$ & $10(17.2)$ & 2.0 \\
\hline
\end{tabular}

a. Data were available for 88 individuals (23 frequent and 65 single attenders). b. Data were available for 79 individuals ( 21 frequent and 58 single attenders).

\begin{tabular}{|c|c|c|c|c|}
\hline \multirow[t]{2}{*}{ Table 2} & \multicolumn{4}{|c|}{$\begin{array}{l}\text { Comparison of the primary diagnoses of the two } \\
\text { groups of patients presenting to the accident } \\
\text { and emergency department }\end{array}$} \\
\hline & & \multicolumn{2}{|c|}{$n(\%)$} & \multirow[b]{2}{*}{$\chi^{2}$} \\
\hline \multicolumn{2}{|c|}{ Diagnosis } & $\begin{array}{l}\text { Frequent } \\
\text { attenders }^{\mathrm{a}}\end{array}$ & $\begin{array}{l}\text { Single } \\
\text { attenders }^{b}\end{array}$ & \\
\hline \multicolumn{2}{|c|}{ Anxiety/neurotic disorders } & $2(8.3)$ & $9(12.5)$ & 0.31 \\
\hline \multicolumn{2}{|c|}{ Bipolar affective disorder } & $2(8.3)$ & $5(6.9)$ & 0.31 \\
\hline \multicolumn{2}{|c|}{ Personality disorders } & $2(8.3)$ & $10(13.9)$ & 0.51 \\
\hline \multicolumn{2}{|c|}{ Alcohol/substance misuse } & $3(12.5)$ & $8(11.1)$ & 0.03 \\
\hline \multicolumn{2}{|c|}{ Depressive disorders } & $8(33.3)$ & $30(41.7)$ & 0.52 \\
\hline \multicolumn{2}{|c|}{ Schizophrenia } & $7(29.2)$ & $8(11.1)$ & $4.45^{*}$ \\
\hline \multicolumn{2}{|l|}{ None } & $0(0)$ & $2(2.8)$ & 0.68 \\
\hline
\end{tabular}

a. $n=24$.

b. $n=72$

${ }^{\star} P<0.05$.

Table 3 Comparison of psychiatric service use by the frequent and single attenders at the accident and emergency department

\begin{tabular}{lccccc} 
& \multicolumn{2}{c}{$n(\%)$} & & \\
\cline { 2 - 4 } Psychiatric service & $\begin{array}{c}\text { Frequent } \\
\text { attenders }^{\mathrm{a}}\end{array}$ & $\begin{array}{c}\text { Single } \\
\text { attenders }^{\mathrm{b}}\end{array}$ & $\chi^{2}$ & $P$ \\
\hline In-patients & $15(62.5)$ & $27(37.5)$ & 4.6 & 0.03 \\
\hline Day hospital attenders & $5(20.8)$ & $5(6.9)$ & 3.7 & 0.05 \\
\hline Out-patients & $11(45.8)$ & $36(50)$ & 0.13 & 0.72
\end{tabular}

a. $n=24$.

b. $n=72$.

admission to the psychiatric unit - on average, 4.5 days (s.d.=9.2), compared with 6.9 days for single attenders (s.d.=23.7). The median time, in days, was 0 for both patient groups. There was no statistically significant difference in the mean ranks for frequent and single attenders (Mann-Whitney $U=217, P=0.20$ ).

A total of 60 patients (17 frequent and 43 single attenders) were identified as having been admitted to the acute psychiatric unit at least once during the 10-year period between 1997 and 2007. Of these, frequent attenders had more admissions compared with single attenders (Mann-Whitney $U=232, P=0.021$ ). The mean number of 
admissions among frequent attenders was 8.2 (s.d. = 10.7, median 6), whereas the mean for single attenders was 4.9 (s.d. = 10.3, median 1).

Frequent attenders were found to be admitted for a mean of 15.7 days (s.d. $=12.6$ ) compared with 20.3 days (s.d. $=34.3$ ) for single attenders. The mean rank of duration of admission was 31.6 for frequent attenders (median 18), whereas that for single attenders was 30.1 (median 11). This difference was not statistically significant (Mann-Whitney $\mathrm{U}=346.5, P=0.76)$.

\section{Discussion}

We found that frequent attenders represented a small proportion of patients who attended the A\&E department seeking psychiatric care. However, these frequent attenders accounted for a substantial proportion of such attendances. These findings are similar to those of Pasic et al. ${ }^{5}$ We also found that frequent attenders had longer psychiatric histories, higher rates of schizophrenia, and were more likely to be prescribed antipsychotics, but had equal gender distribution as compared with single attenders. Chaput \& Lebel $^{6}$ reported similar findings in their study of similar patient population.

We found frequent attenders to have higher rates of acute psychiatric hospital admissions, which on average were shorter in duration compared with those of single attenders. Carpenter $e t a l^{7}$ made similar observations in their study of multiple admissions to an urban psychiatric centre in New York. Additionally, we found that the mean interval between hospital discharges and A\&E presentations was on average shorter for frequent attenders compared with single attenders. As the A\&E department serves as an entry point for in-patient admission, the higher attendance to $A \& E$ by patients who also have high rates of psychiatric admissions is to be expected. The higher rate of psychiatric admissions is also consistent with the higher rates of schizophrenia diagnosis in this group, as exacerbation of such severe and enduring mental illness is likely to meet criteria for in-patient admission. However, the therapeutic effectiveness of such short interventions suggests the need for review of management strategy to prevent 'revolving door' admissions. When admission is necessary, perhaps lengthening the in-patient stay may better prepare patients for discharge.

Greater use of hospital-based services by these individuals suggests that their needs are not adequately met by their management plans in the community. As repeated attendance to the $\mathrm{A} \& \mathrm{E}$ department for psychiatric care is not an optimal treatment strategy, and hospital beds are at a massive premium, it is imperative for mental health services to carefully evaluate the quality, appropriateness and effectiveness of available community services to better target the needs of frequent users of hospital-based services. ${ }^{3}$ Well-developed community-oriented strategies, such as modified intensive case management and outreach programmes, which have been shown to reduce the heavy use of psychiatric in-patient services, ${ }^{8}$ could also help limit frequent attenders' visits by reducing social instability and increasing treatment adherence. ${ }^{6}$

\section{Study limitations}

The small sample size, short period examined and the retrospective design with consequent use of unverified and often incomplete information from hospital records may be regarded as limitations of this study. These methodological differences could explain the reason for the absence of lower scores on measures of social stability (living situation, marital and employment status) among frequent attenders as reported by Sullivan et al. ${ }^{4} \mathrm{~A}$ good predictor of admission not examined in this study is the closeness of A\&E attendances by frequent attenders - this can also be construed as a weakness to this study.

\section{About the authors}

Eugene F. Okorie is Senior Registrar in General Adult Psychiatry, Colm McDonald is Professor of Psychiatry and Consultant Psychiatrist, Department of Psychiatry, National University of Ireland, Galway, Brendan Dineen is Researcher, Department of Medical Informatics and Medical Education, National University of Ireland, Galway.

\section{References}

1 Bassuk E, Gerson S. Chronic crisis patients: a discrete clinical group. Am J Psychiatry 1980; 137: 1513-7.

2 Breslow RE, Erickson BJ, Cavanaugh KC. The psychiatric emergency service: where we've been and where we're going. Psychiatr Q 2000; 71: $101-21$.

3 Arfken C, Zeman L, Yeager L, White A, Mischel E, Amirsadri A. Case control study of frequent visitors to an urban psychiatric service. Psychiatr Serv 2004; 55: 295-301.

4 Sullivan PF, Bulik CM, Forman SD, Mezzich JE. Characteristics of repeat users of a psychiatric emergency service. Hosp Community Psychiatry 1993; 44: 376-80

5 Pasic J, Russo J, Roy-Byre P. High utilizers of psychiatric emergency services. Psychiatr Serv 2005; 56: 678-84.

6 Chaput YJA, Lebel MJ. Demographic and clinical profiles of patients who make multiple visits to psychiatric emergency services. Psychiatr Serv 2007; 58: 335-41.

7 Carpenter MD, Mulligan JC, Bader IA, Meinzer AE. Multiple admissions to an urban psychiatric centre: a comparative study. Hosp Community Psychiatry 1985; 36: 1305-8.

8 Quinlivan R, Hough R, Crowell A, Beach C, Hofsteller R, Kenworthy K. Service utilization and costs of care for severely mentally ill clients in an intensive case management program. Psychiatr Serv 1995; 46: 365-71. 